\title{
The correlation of pulmonary arterial hypertension with late recurrence of paroxysmal atrial fibrillation after catheter ablation
}

\author{
Yan-Qun Zhang', Fei-Long Zhang ${ }^{2}$, Wei-Wei Wang' ${ }^{2}$, Xue-Hai Chen ${ }^{2}$, Jian-Hua Chen ${ }^{2}$, Liang-Long \\ Chen $^{2}$ \\ ${ }^{1}$ Deparment of Medical Ultrasonics, Xiamen Branch, Zhongshan Hospital, Fudan University, Xiamen 361000, China; ${ }^{2}$ Department of Cardiology, \\ Fujian Medical University Union Hospital, Fuzhou 350001, China \\ Contributions: (I) Conception and design: LL Chen, FL Zhang; (II) Administrative support: LL Chen, FL Zhang; (III) Provision of study materials or \\ patients: JH Chen; (IV) Collection and assembly of data: YQ Zhang; (V) Data analysis and interpretation: YQ Zhang; (VI) Manuscript writing: All \\ authors; (VII) Final approval of manuscript: All authors. \\ Correspondence to: Fei-Long Zhang, MD, PhD. Department of Cardiology, Fujian Medical University Union Hospital, No. 29 of Xinquan Street, \\ Gulou District, Fuzhou, 350001, China. Email: feilongzhang@21cn.com.
}

Background: Paroxysmal atrial fibrillation (PAF) is one of the most common clinical arrhythmias. Although radiofrequency catheter ablation (RFCA) for the treatment of atrial fibrillation has continuously matured and developed in recent years, some patients treated with RFCA continued to have atrial fibrillation recurrence, and the recurrence rate was high. Determining indicators to predict the recurrence of PAF after RFCA is significantly important for improving the surgical success rate and guiding clinical work. This study aimed to investigate the influence of pulmonary arterial hypertension (PAH) on the late recurrence of PAF after RFCA.

Methods: A total of 300 patients with PAF, who underwent RFCA for the first time at the Department of Cardiology of Fujian Union Medical College Hospital from January 2013 to October 2016, were retrospectively studied. These patients were regularly followed-up from 3 months at least to 3 years and clinical data were collected. In order to observe the 100 PAF patients with PAH were assigned into the observation group, and $200 \mathrm{PAF}$ patients without $\mathrm{PAH}$ were assigned as the control group. PAH and its related clinical characteristics were evaluated by univariate analysis of variance (ANOVA) and logistic regression analysis.

Results: The follow-up results revealed that 34 patients had early recurrence, and the early arrhythmia recurrence rate was $11.3 \%$. Furthermore, 22 patients had late recurrence, including 19 patients with atrial fibrillation and three patients with atrial flutter; and the late recurrence rate was $7.3 \%$. The univariate ANOVA revealed that $\mathrm{PAH}(\mathrm{P}=0.001)$, early recurrence $(\mathrm{P}=0.014)$ and Left atrial diameter $(\mathrm{LAD})(\mathrm{P}=0.023)$ had significant effects on late recurrence after PAF ablation. Furthermore, logistic regression analysis revealed that $\mathrm{PAH}(\mathrm{P}=0.049$, OR $=1.053$, 95\% CI: 1.000-1.109) was independently correlated to late recurrence of PAF.

Conclusions: PAH is a predictive factor for late recurrence of PAF after RFCA.

Keywords: Atrial fibrillation (AF); pulmonary arterial hypertension (PAH); radiofrequency catheter ablation (RFCA); late recurrence

Submitted Nov 28, 2017. Accepted for publication Mar 21, 2018.

doi: $10.21037 /$ jtd.2018.04.92

View this article at: http://dx.doi.org/10.21037/jtd.2018.04.92 


\section{Introduction}

Atrial fibrillation (AF) is one of the most common clinical arrhythmia diseases that affect the health of the elderly at present and data in 2013 revealed that AF occurred in approximately 33.5 million patients in the world (1-3). In 2004, Zijiang Zhou et al. published the first largescale epidemiological research results for AF in China, and the data revealed that the total morbidity of AF in China reached $0.61 \%$ significantly higher than the general population morbidity of $0.4 \%$ (4). Moreover, there was a tendency for morbidity to increase annually. The incidence of $\mathrm{AF}$ in a population of over 80 years old has reached 6-8\% $(5,6)$. AF was a common disease that led to clinical death and disability, which greatly increasing socio-economic and medical burden (7-9). In recent years, radiofrequency catheter ablation (RFCA) has become the most promising prospective treatment for $\mathrm{AF}$, due to its advantages of minimal invasiveness and safety. Furthermore, RFCA surgical success rate can often reach up to $70-80 \%$ in experienced electrophysiological centers. In addition, it can reduce the incidence of complications by $55 \%$, and reduce mortality by $54 \%(10,11)$. However its postoperative recurrence rate can reach as high as $20-60 \%$, although ablation methods have continuously been improved and matured (12). Therefore, determining factors that affect the recurrence of AF after ablation would contribute to clinicians in choosing better surgical indications for treatment, and is significantly important for improving the surgical successful rate and guiding clinical work.

Pulmonary arterial hypertension (PAH) refers to a vascular disease that mainly involves small pulmonary arteries. The main pathophysiological change is that pulmonary artery pressure gradually increases with disease progression, causing changes in cardiac hemodynamics, pulmonary vascular remodeling, followed by right ventricular hypertrophy and heart function decline. The secondary myocardial remodeling of the right atrium induced by long-term pressure and capacity overload would be the basis of atrial arrhythmia. Therefore, we consider that $\mathrm{PAH}$ is the affecting factor for AF recurrence after RFCA. The gold standard for the diagnosis of PAH in the ESC is that the mean pulmonary artery pressure is of greater than $25 \mathrm{mmHg}$ measured by right heart catheterization at sea level. Pulmonary arterial pressure (PAP) measured by the right heart catheter is invasive, costly and may lead to complications. Therefore, echocardiography mainly through measure the tricuspid regurgitation pressure to estimate pulmonary artery pressure (PAP) is more easily become routine clinically. The grading standards of PAP measure by echocardiography: normal PAP less than $30 \mathrm{mmHg}$, pressure 31 to $50 \mathrm{mmHg}$ belong to mild $\mathrm{PAH}$, moderate $\mathrm{PAH}$ at wedge 51 to $70 \mathrm{mmHg}$ and severe $\mathrm{PAH}$ greater than $70 \mathrm{mmHg}$. PAP was measured by echocardiography in this study.

\section{Methods}

\section{Selection criteria}

From January 2013 to October 2016, 300 consecutive patients were referred to our center for RFCA due to symptomatic paroxysmal AF. Paroxysmal AF was defined as the occurrence of two or more episodes of AF during the previous year, and all of which terminated spontaneously within one week. Patients were excluded from the study based on the following criteria: (I) patients $<18$ or $>80$ years old; (II) patients with New York Heart Association (NYHA) functional class IV; (III) preoperative transesophageal echocardiography prompted left atrium and/or left atrial appendage with unmachinated thrombus; (IV) patients with hyperthyroidism; (V) patients who have had a concurrent cerebrovascular accident or other neurological diseases within three months; (VI) patients complicated with severe respiratory disease, severe immune system diseases, severe liver and kidney dysfunction, severe metabolic disorders, or malignant tumors; (VII) patients who underwent surgery within three months; (VIII) patients who have a tendency to bleed; (IX) patients who refused to undergo surgery; (X) patients who post-operatively cannot adhere two months of anticoagulant therapy; (XI) patients with non-pulmonary venous origin of AF; (XII) patients with incomplete clinical data or lost to follow-up.

The study protocol was approved by the local Ethics Committee (The number of the ethic approval is Fujian Medical University Union Hospital 2017KY010.), and a written informed consent was obtained from all patients.

\section{Radiofrequency ablation technique}

Patients were placed in the supine position, conventional disinfection towels were placed, local anesthesia was induced with $1 \%$ lidocaine. Puncture was performed at the left subclavian vein, and a vein sheath was placed, which was guided to the coronary sinus. The septum interval was punctured through the right femoral vein twice, and 
$100 \mathrm{u} / \mathrm{kg}$ of normal heparin was injected into the two sheaths to the left atrium. Non-selective angiography was performed on the left atrium and pulmonary vein. A $15-\mathrm{mm}$ single-loop lead catheter was placed into the left atrium, the left upper and lower pulmonary veins were determined, and the upper and lower right pulmonary veins were electrical connected to the left atrium. Then, the catheter for cryopreserved saline was guided into the left atrium. Next, bilateral pulmonary vein vestibular linear ablation was performed to isolate the pulmonary vein. The radiofrequency ablation end-point was the disconnection of the pulmonary vein with the atrium, and the atrial blowout stimulation failed to induce $<30$ seconds of rapid atrial arrhythmia. Radiofrequency energy was delivered at a maximal temperature of $43{ }^{\circ} \mathrm{C}$, a maximal power of $35 \mathrm{~W}$, and a discharge of saline irrigation rate of $17-20 \mathrm{~mL} / \mathrm{min}$. Postoperative wound pressure was applied to stop the bleeding, and sterile gauze was used for pressure bandage.

\section{The methodology of measuring pulmonary pressure}

In this study, PAP was estimated by tricuspid regurgitation pressure measured by continuous wave Doppler combined with right atrial pressure estimated by ultrasound. Specific measurement methods are as follows: simplified Bernouli equation $\Delta \mathrm{P}=4 \mathrm{~V}_{\mathrm{TR}}^{2}$, the tricuspid regurgitation peak velocity can be converted into tricuspid valve transvalvular pressure, right ventricular systolic pressure can be equal to PAP in the absence of right ventricular outflow obstruction, and right atrial systolic pressure is estimated by right ventricular size and the diameter of the inferior vena cava measured by ultrasound, usually equal to $10 \mathrm{mmHg}(1.33 \mathrm{kPa})$, so ultimately $\mathrm{PAP}=\Delta \mathrm{P}+\mathrm{RAP}=4 \mathrm{~V}_{\mathrm{TR}}^{2}+10 \mathrm{mmHg}$. Pressure greater than $31 \mathrm{mmHg}$ is defined as $\mathrm{PAH}$, pressure 31 to $50 \mathrm{mmHg}$ belong to mild $\mathrm{PAH}$, moderate $\mathrm{PAH}$ at wedge 51 to $70 \mathrm{mmHg}$ and severe $\mathrm{PAH}$ greater than $70 \mathrm{mmHg}$.

\section{Follow-up and data collection}

All patients were routinely monitored with 24-hour ECG after radiofrequency ablation immediately. Medications such as warfarin were continued based on the discretion of the physician, and antiarrhythmics were stopped at two months after ablation.

Patients were routinely followed-up from 3 months at least to 3 years by telephone at least once a month after ablation, and weekly with regular echocardiography (ECG) and opportunistic ECG upon onset of the symptom. Then, 24-hour Holter monitoring and ECG examinations were performed in all patients every three months after the ablation. The late recurrence of AF was defined as any atrial tachycardia that lasted for more than 30 seconds on 24-hour Holter monitoring or documented on 12-lead ECG after a 3 -month blanket period of operation, and early recurrence was within three months. Patients were divided into two groups on the basis of late recurrence: maintained sinus rhythm (SR group) and recurrence of AF (AF group).

By reviewing the medical history, collect the following relevant clinical data: (I) clinical data before ablation includes the gender, age, the course of AF, history of hypertension, diabetes, hyperlipemia, hypertensive heart disease, coronary artery disease, dilated cardiomyopathy, valvular heart disease and grown-up with congenital heart disease which mainly refers to atrial septal defect and patent foramen ovale; (II) related laboratory test results before ablation include high density lipoprotein cholesterol, low density lipoprotein cholesterol, total cholesterol, triglyceride; (III) echocardiographic examination data include left ventricular end diastolic diameter, left ventricular end-systolic dimension, left atrial diameter, left ventricular ejection fraction, E/E' and pulmonary arterial pressure.

\section{Statistical analysis}

Statistical analysis was performed utilizing SPSS 19.0 software. Continuous data were expressed as mean \pm standard deviation (SD). Categorical data were expressed as absolute numbers or percentages. Differences between the groups (SR and AF groups) were tested using independent sample $t$-test and chi-square test. The correlations between $\mathrm{AF}$ recurrence after RFCA and its predictors were assessed by multivariable logistic regression analysis. Statistical significance was assumed at $\mathrm{P}<0.05$.

\section{Results}

\section{Baseline characteristics}

A total of 300 patients were treated with RFCA for AF and were followed up. The results are shown in Table 1. Among them, 244 patients had sinus rhythm without any AF recurrence, 34 (11.3\%) patients had AF early recurrence, and $22(7.3 \%)$ had late recurrence.

Furthermore, among these patients, 174 (58\%) were male and 126 (42\%) were female. The mean age of these 
Table 1 The baseline characteristics of the patients were included

\begin{tabular}{lc}
\hline Characteristic & $\bar{x} \pm \mathrm{s} / \mathrm{n}(\%)$ \\
\hline Gender (male/female) & $174(58.0) / 126(42.0)$ \\
Age (years) & $59.66 \pm 9.702$ \\
Course of atrial fibrillation (months) & $57.05 \pm 74.05$ \\
Follow-up time (months) & $22.31 \pm 12.60$ \\
HT & $145(48.3)$ \\
Diabetes & $53(17.7)$ \\
HLP & $100(33.3)$ \\
HTHD & $65(21.7)$ \\
CAD & $18(6.0)$ \\
DM & $12(4.0)$ \\
VHD & $28(9.3)$ \\
GUCH (ASD or PFO) & $12(4.0)$ \\
Early recurrence of AF & $34(11.3)$ \\
Late recurrence of AF & $22(7.3)$ \\
\hline
\end{tabular}

HT, hypertension; HLP, hyperlipemia; HTHD, hypertensive heart disease; CAD, coronary artery disease; DM, dilated cardiomyopathy; VHD, valvular heart disease; GUCH, grown-up with congenital heart disease; ASD, atrial septal defect; PFO, patent foramen ovale.

patients was $59.66 \pm 9.702$ years old, and the mean follow-up time was $22.31 \pm 12.60$ months.

\section{Univariate analysis}

There patients were divided into two groups on the basis of late recurrence: maintained sinus rhythm (SR group) and recurrence of $\mathrm{AF}$ ( $\mathrm{AF}$ group). The results of the univariate analysis were listed in Tables 2 and 3. The univariate analysis revealed that early recurrence $(\mathrm{P}=0.014)$, LA diameter $(\mathrm{P}=0.023)$ and pulmonary arterial pressure $(\mathrm{P}=0.001)$ were important factors that affect the late recurrence of $\mathrm{AF}(\mathrm{P}<0.05)$. Patients in the AF group had higher LA diameters $(38.30 \pm 5.01$ vs. $35.83 \pm 5.55 \mathrm{~mm}, \mathrm{P}=0.023)$ and higher pulmonary arterial pressure $(31.86 \pm 6.21$ vs. $26.33 \pm 9.46 \mathrm{mmHg})$, compared to patients in the SR group; and the difference was statistically significant.

\section{Predicting the late recurrence of $A F$}

The results of the logistic regression analysis on the recurrence of $\mathrm{AF}$ are summarized in Table 4. Univariate
Table 2 Categorical data follow-up in the SR group or the AF group

\begin{tabular}{lccc}
\hline Characteristic & SR group & AF group & P \\
\hline Case & 278 & 22 & \\
Gender (male/female) & $164 / 114$ & $10 / 12$ & 0.216 \\
HT & $135(48.6)$ & $10(45.5)$ & 0.779 \\
Diabetes & $51(18.3)$ & $2(9.1)$ & 0.273 \\
HLP & $91(32.7)$ & $9(40.9)$ & 0.434 \\
HTHD & $62(22.3)$ & $3(13.6)$ & 0.342 \\
CAD & $18(6.5)$ & $0(0.0)$ & 0.218 \\
DM & $12(4.3)$ & $0(0.0)$ & 0.320 \\
VHD & $26(9.4)$ & $2(9.1)$ & 0.968 \\
GUCH (ASD or PFO) & $10(3.6)$ & $2(9.1)$ & 0.206 \\
Early recurrence of AF & $28(10.1)$ & $6(27.3)$ & $0.014^{*}$ \\
\hline
\end{tabular}

*, $\mathrm{P}<0.05$. HT, hypertension; HLP, hyperlipemia; HTHD, hypertensive heart disease; CAD, coronary artery disease; DM, dilated cardiomyopathy; VHD, valvular heart disease; $\mathrm{GUCH}$, grown-up with congenital heart disease; ASD, atrial septal defect; PFO, patent foramen ovale.

analysis revealed that early recurrence, left atrial diameter (LAD) and PAH were associated with late recurrence AF. Furthermore, multivariate analysis identified $\mathrm{PAH}$ as an independent predictor of $\mathrm{AF}$ recurrence [odds ratio $(\mathrm{OR})=1.053,95 \%$ confidence interval $(\mathrm{CI}): 1.000-1.109$, $\mathrm{P}=0.049]$.

\section{Discussion}

\section{Pulmonary arterial bypertension}

In the present study, we reviewed the clinical data on the ablation performed for PAF in our hospital over the past four years. The relationship between pulmonary hypertension and late recurrence after RFCA was analyzed through statistical methods. Results revealed that preoperative $\mathrm{PAH}$ was one of the independent risk factors for late recurrence after RFCA in patients with PAF. The mechanism for the recurrence may be the increase in pulmonary arterial pressure, which resulted in the gradual increase in right ventricular pressure load and capacity load. As the disease progresses, right ventricular myocardium secondary compensatory hypertrophy and expansion occurs, and right ventricular pressure increases, resulting in right ventricular secondary myocardium remodeling, 
Table 3 Continuous data follow-up in the SR group or the AF group

\begin{tabular}{lccc}
\hline Characteristic & SR group & AF group & $P$ \\
\hline Case & 278 & 22 & \\
Age (years) & $59.41 \pm 9.86$ & $62.86 \pm 6.83$ & 0.108 \\
BMI (kg/m²) & $25.37 \pm 18.38$ & $24.03 \pm 2.40$ & 0.733 \\
Course (months) & $55.29 \pm 73.15$ & $79.36 \pm 83.30$ & 0.142 \\
HDL-C (mmol/L) & $1.20 \pm 0.33$ & $1.21 \pm 0.39$ & 0.857 \\
LDL-C (mmol/L) & $2.78 \pm 0.92$ & $3.04 \pm 0.68$ & 0.197 \\
TC (mmol/L) & $4.37 \pm 1.05$ & $4.64 \pm 0.77$ & 0.246 \\
TG (mmol/L) & $1.83 \pm 5.65$ & $1.59 \pm 0.63$ & 0.840 \\
LVEDD (mm) & $47.90 \pm 4.59$ & $46.90 \pm 4.11$ & 0.324 \\
LVESD (mm) & $31.06 \pm 17.77$ & $29.59 \pm 2.90$ & 0.699 \\
LAD (mm) & $35.83 \pm 5.55$ & $38.30 \pm 5.01$ & $0.023^{\star}$ \\
LVEF (\%) & $66.57 \pm 7.76$ & $66.61 \pm 4.10$ & 0.974 \\
E/E & $7.22 \pm 2.36$ & $8.66 \pm 3.04$ & 0.226 \\
PAP (mmHg) & $26.33 \pm 9.46$ & $31.86 \pm 6.21$ & $0.001^{*}$ \\
\hline
\end{tabular}

*, $\mathrm{P}<0.05$. BMI, body mass index; HDL-C, high density lipoprotein cholesterol; LDL-C, low density lipoprotein cholesterol; TC, total cholesterol; TG, triglyceride; LVEDD, left ventricular end diastolic diameter; LVESD, left ventricular end-systolic dimension LAD, left atrial diameter; LVEF, left ventricular ejection fraction; E, early filling; $\mathrm{E}$, early diastolic; PAP, pulmonary arterial pressure.

and right atrial enlargement. After long-term right atrial enlargement, myocardial cell interstitial fibrosis is induced, intercellular link changes, and atrial arrhythmia occurs. AF can be maintained based on atrial matrix reconstruction (13). At the same time, due to the persistent onset of AF, atrial systolic function is gradually decreased and intraventricular pressure is increased, causing pulmonary circulation pressure to increase. Therefore, PAH can be maintained and developed, which was a vicious cycle (14). Few studies have been conducted on the correlation between $\mathrm{PAH}$ and the recurrence of PAF after RFCA, which needs to be studied further through multicenter studies with large sample data.

\section{Early recurrence}

Studies have shown that $(15,16)$ early recurrence is a predictor of late recurrence after RFCA. In terms of its mechanism, it should be considered that early recurrence
Table 4 Predictors of AF late recurrence

\begin{tabular}{lccc}
\hline Characteristic & $P$ & Odds ratio & $\begin{array}{c}95 \% \text { confidence } \\
\text { interval }\end{array}$ \\
\hline PAP & $0.049^{*}$ & 1.053 & $1.000-1.109$ \\
LAD & 0.103 & 1.066 & $0.987-1.152$ \\
Early recurrence of AF & 0.067 & 0.375 & $0.131-1.071$ \\
\hline
\end{tabular}

*, P<0.05. PAP, pulmonary arterial pressure; LAD, left atrial diameter.

may prevent the recurrence of atrial tissue after ablation remodeling, thereby increasing the possibility of late recurrence. However, many scholars (17) believed that the early recurrence of various arrhythmias was irrelevant to late recurrence, which was mostly a temporary phenomenon. The mechanism of early recurrence may be that local tissue edema occurred at the early period after RFCA, and resulted to shorter effective atrial refractory period or increased ectopic self-discipline abnormalities, which is generally self-delayed healing (18). The rate of delayed healing in this study was $35.3 \%$, which included 12 patients, in which their atrial arrhythmia events that occurred at early stage gradually disappeared three months after RFCA. Therefore, early recurrence does not lead to failure of RFCA. In other words, patients with early recurrence should be given delayed healing time in clinic.

\section{Left atrial diameter}

LAD is one of the independent risk factors for the late recurrence of AF, which has been confirmed (19). The main mechanism is that the left atrial enlargement caused the reentry pathway to increase and induce interstitial fibrosis, subsequently slowing down the conduction rate. Moreover, the increase in left atrial load induced atrial wall compensatory thickening. Therefore, the refractory period of the atrium shortened and the conduction time was extended. The role of structural remodeling and electrical remodeling induced and maintained the occurrence of $\mathrm{AF}$.

\section{Conclusions}

Patients with late recurrence AF had significantly higher pulmonary arterial pressure, compared to patients with SR. $\mathrm{PAH}$ before RFCA was an independent predictor for the late recurrence of AF after ablation. Avoiding PAF with $\mathrm{PAH}$ in RFCA was significantly important for improving the surgical successful rate and guiding clinical work. 


\section{Acknowledgements}

Funding: Fujian Province Natural Foundation (2014J01326), Fujian Province Medical Innovation Project (2016-CX-17).

\section{Footnote}

Conflicts of Interest: The authors have no conflicts of interest to declare.

Ethical Statement: This study was conducted in accordance with the declaration of Helsinki. This study was conducted with approval from the Ethics Committee of Fujian Medical University Union Hospital in number 2017KY010. Written informed consent was obtained from all participants.

\section{References}

1. Fuster V, Rydén LE, Cannom DS, et al. 2011 ACCF/ AHA/HRS focused updates incorporated into the ACC/ AHA/ESC 2006 Guidelines for the management of patients with atrial fibrillation: a report of the American College of Cardiology Foundation/American Heart Association Task Force on Practice Guideline. J Am Coll Cardiol 2011;57:e101-98.

2. Chugh SS, Havmoeller R, Narayanan K, et al. Worldwide epidemiology of atrial fibrillation: a Global Burden of Disease 2010 Study. Circulation 2014;129:837-47.

3. Krijthe BP, Kunst A, Benjamin EJ, et al. Projections on the number of individuals with atrial fibrillation in the European Union, from 2000 to 2060. Eur Heart J 2013;34:2746-51.

4. Zhou ZQ, Hu DY, Chen J, et al. An epidemiological survey of atrial fibrillation in China. Zhonghua Nei Ke Za Zhi 2004;43:491-4.

5. Wolf PA, Abbott RD, Kannel WB, et al. Atrial fibrillation as an independent risk factor for stroke: the Framingham Study. Stroke 1991;22:983-8.

6. Furberg CD, Psaty BM, Manolio TA, et al. Prevalence of atrial fibrillation in elderly subjects (the Cardiovascular Health Study). Am J Cardiol 1994;74:236-41.

7. Wolf PA, Dawber TR, Thomas HE Jr, et al. Epidemiologic assessment of chronic atrial fibrillation and risk of stroke the fiamingham Study. Neurology 1978;28:973-7.

8. Seiler J, Stevenson WG. Atrial fibrillation in congestive heart failure. Cardiol Rev 2010;18:38-50.

9. Ott A, Breteler MM, de Bruyne MC, et al. Atrial fibrillation and dementia in a population-based study. The Rotterdam Study. Stroke 1997;28:316-21.
10. Cappato R, Calkins H, Chen SA, et al. Updated worldwide survey on the methods, efficacy, and safety of catheter ablation for human atrial fibrillation. Circ Arrhythm Electrophysiol 2010;3:32-8.

11. Pappone C, Rosanio S, Augello G, et al. Mortality, morbidity, and quality of life after circumferential pulmonary vein ablation for atrial fibrillation: outcomes from a controlled nonrandomized long-term study. J Am Coll Cardiol 2003;42:185-97.

12. Takahashi Y, Takahashi A, Kuwahara T, et al. Clinical characteristics of patients with persistent atrial fibrillation successfully treated by left atrial ablation. Circ Arrhythm Electrophysiol 2010;3:465-71.

13. Allessie M, Ausma J, Schotten U, et al. Electrical, contractile and structural remodeling during atrial fibrillation. Cardiovasc Res 2002;54:230-46.

14. Medi C, Kalman JM, Ling LH, et al. Atrial electrical and structural remodeling associated with longstanding pulmonary hypertension and right ventricular hypertrophy in humans. J Cardiovasc Electrophysiol 2012;23:614-20.

15. Cai L, Yin Y, Ling Z, et al. Predictors of late recurrence of atrial fibrillation after catheter ablation. Int $\mathrm{J}$ Cardiol 2013;164:82-7.

16. Lee SH, Tai CT, Hsieh MH, et al. Predictors of early and late recurrence of atrial fibrillation after catheter ablation of paroxysmal atrial fibrillation. J Interv Card Electrophysiol 2004;10:221-6.

17. Seow SC, Lim TW, Koay CH, et al. Efficacy and late recurrences with wide electrical pulmonary vein isolation for persistent and permanent atrial fibrillation. Europace 2007;9:1129-33.

18. Pappone C, Santinelli V. The who, what, why, and howto guide for circumferential pulmonary vein ablation. $\mathrm{J}$ Cardiovasc Electrophysiol 2004;15:1226-30.

19. Dogan A, Kahraman H, Ozturk M, et al. P wave dispersion and left atrial appendage function for predicting recurrence after conversion of atrial fibrillation and relation of $\mathrm{p}$ wave dispersion to appendage function. Echocardiography 2004;21:523-30.

Cite this article as: Zhang YQ, Zhang FL, Wang WW, Chen XH, Chen JH, Chen LL. The correlation of pulmonary arterial hypertension with late recurrence of paroxysmal atrial fibrillation after catheter ablation. J Thorac Dis 2018;10(5):27892794. doi: $10.21037 /$ jtd.2018.04.92 